Ann. Génét. Sél. anim., I979, 11 (4), 4I3-424.

\title{
Estimation des paramètres génétiques de la taille de portée des agnelles Lacaune après fécondation sur œstrus naturel et induit
}

\author{
L. BODIN
}

Station d'amélioration génétique des animaux, I.N.R.A.

Centre de Recherches de Toulouse, B.P. I2, 31320 Castanet-Tolosan

\section{Résumé}

Les paramètres génétiques de la taille de portée avec fécondation sur œstrus naturel ou sur œstrus induit (après utilisation d'éponge vaginale imprégnée de F.G.A., et injection de PMSG) ont été analysés sur 9 I Io agnelles Lacaune de $\mathrm{r} 70$ troupeaux laitiers. La prolificité sur ostrus induit $(\mathrm{I}, 4 \mathrm{I})$ est très significativement supérieure à la prolificité sur œstrus naturel $(\mathrm{I}, \mathrm{I} 8)$. Les effets de l'âge à la mise-bas et de l'époque d'agnelage sur la prolificité ne sont pas significatifs.

L'héritabilité de la prolificité, estimée par analyse de variance des demi-sœurs de père, est significativement différente de zéro $\left(h^{2}=0.064\right)$ pour la " prolificité induite ", ce qui n'est pas le cas pour la "prolificité naturelie " $\left(h^{2}=0.032\right)$. La corrélation génétique entre ces deux types de performances $(v g=0.33)$ laisse supposer que " prolificité naturelle " et "prolificité induite " sont deux caractères contrôlés en partie par des gènes différents.

A la suite de ces résultats, nous présentons trois situations classiques d'utilisation des traitements hormonaux dans les structures françaises et nous indiquons leurs implications dans l'élaboration de schémas de sélection sur les caractères de reproduction.

\section{Introduction}

Dans le cadre d'un programme de sélection, l'insémination artificielle (I.A.) est un outil indispensable pour mettre en place le programme d'accouplement nécessaire à la production de filles d'un même père dans plusieurs élevages et pour faciliter la diffusion du progrès génétique (FoULLEy et BIBÉ, I979).

Actuellement 1'I.A. n'est pratiquement effectuée qu'après induction de l'œstrus par traitement hormonal. Or, ce traitement modifie les performances de reproduction, ce qui pose des problèmes quant à l'estimation de la valeur génétique des reproducteurs. En ce qui concerne la prolificité avec et sans traitement hormonal, on peut considérer qu'il s'agit d'un même caractère s'exprimant dans deux milieux différents ou de deux caractères plus ou moins liés génétiquement, mais dans les 
deux cas il convient de calculer les paramètres génétiques de ces deux prolificités afin afin d'estimer les réponses génétiques attendues en sélectionnant directement ou indirectement sur l'un des deux critères ou sur les deux à la fois.

Dans ce but, une expérimentation a été programmée dans les troupeaux de brebis Lacaune à viande en rythme accéléré de reproduction avec " maîtrise " des œestrus sur la moitié de chaque descendance intra élevage (Bodin et al., I979). Pour compléter cette expérimentation, nous avons cherché à utiliser les données disponibles dans les élevages de brebis laitières Lacaune qui participent au schéma de testage des béliers sur la production laitière, puisque la moitié environ de ces éleveurs pratiquent la "maitrise " des œestrus sur la totalité de leurs agnelles. Cette étude concerne donc l'analyse a posteriori de la prolificité des agnelles "Lacaune lait ". Après avoir étudié les facteurs génétiques de variation de la prolificité naturelle (sur O.N.) et de la prolificité induite (sur O.I.), nous avons estimé les héritabilités de ces deux caractères et leur corrélation génétique.

\section{Matériel animal}

Les données proviennent de I70 troupeaux de race Lacaune contrôlés par deux organismes de sélection (Ovitest et Confédération). Ces troupeaux se classent en deux catégories suivant la méthode de reproduction appliquée aux agneles : dans les uns, toutes les agnelles sont "synchronisées " puis inséminées ou saillies en monte naturelle, dans les autres, il y a absence de traitement hormonal et uniquement monte naturelle.

Nous avons enregistré les mises-bas de I6 753 agnelles nées pendant l'hiver I975-76 et mises à la reproduction à partir de juillet I976. Parmi celles-ci, nous avons conservé uniquement celles ayant un père et une date de naissance connus et celles qui, après traitement hormona1, ont été fécondées par I.A. sur 1'œstrus induit. Il est resté 9 Iro agnelles dont 4 Ig6 fécondées sur O.N. et 49 I 4 fécondées sur O.I. Ėlles représentent au total, $28 \mathrm{r}$ descendances, mais seulement 48 pères ont à la fois des filles fécondées sur O.N. et sur O.I.

Les techniques de synchronisation des œstrus et d'insémination sont celles préconisées par ThImonier et al. (I968), Colas et al. (I973). Eilles comprennent la pose d'une éponge vaginale imprégnée de $40 \mathrm{mg}$ d'acétate de fluorogestone pendant $I_{4}$ jours en moyenne (I 2 à $\mathrm{I} 7$ suivant les élevages), suivie d'une injection de 400 U.I. de PMSG, l'insémination ayant lieu en une ou deux fois selon les résultats de fertilité de l'année antérieure. Dans ce cas et suite à une étude préliminaire sur la durée de gestation, nous considérons que la fécondation a lieu sur l'œstrus induit lorsque l'intervalle dépose de l'éponge - mise-bas est inférieur à 157 jours, avortement exclu.

\section{Facteurs de variation et variables analysées}

Nous avons considéré l'âge de l'agnelage et l'époque de mise-bas comme facteurs non génétiques de variation de la prolificité, et recherché s'il y a une relation entre l'effectif de renouvellement d'un troupeau et la prolificité moyenne 
de ses agnelles. Pour vérifier que l'appartenance des mâles à un schéma de sélection sur les caractères laitiers n'introduit pas une erreur systématique dans l'estimation des paramètres génétiques de la prolificité, nous avons calculé la corrélation entre les index " lait " et les index " prolificité ".

\section{Echantillons du fichier}

Étant donnée la répartition des descendances contrôlées dans les deux catégories d'élevage, il n'est pas possible d'estimer des paramètres génétiques sur l'ensemble des données, aussi avons-nous constitué 6 échantillons différents :

A : Ellevages sans traitement hormonal sur les agnelles: 69 élevages, 4 r 96 agnelles.

B : Élevages avec traitement hormonal sur les agnelles : ror élevages, 4 I94 agnelles.

$\mathrm{C}$ et $\mathrm{D}$ : Ensemble des descendances avec au moins ro filles réparties dans un minimum de 3 élevages.

C : 63 mâles et I 474 agnelles en O.N.

D : 89 mâles et 2 zoI agnelles en O.I.

$\mathrm{E}_{1}$ et $\mathrm{E}_{2}$ : Descendances des 48 béliers ayant au minimum ro filles en $\mathrm{O} . \mathrm{N}$. $\left(\mathrm{E}_{1}=\right.$ I 129 agnelles) et Io filles en O.I. ( $E_{2}=$ I 328 agnelles) réparties chaque fois dans au moins 2 élevages.

Les fichiers $A$ et $B$ permettent l'analyse des facteurs non génétiques de variation de la taille de portée. Les fichiers $\mathrm{C}$ et $\mathrm{D}$ servent à l'estimation des héritabilités et des index prolificité, et les fichiers $\mathrm{E}_{1}$ et $\mathrm{E}_{2}$ sont utilisés pour estimer la corrélation génétique.

\section{Calcul des Index}

Les index prolificité pour apprécier la valeur génétique additive $\mathrm{G}_{i}$ d'un mâle $i$ sont calculés pour les deux types $(\theta=\mathrm{I}, 2)$ de prolificité en exprimant la performance de chaque fille du mâle en écart à la moyenne des performances des agnelles de son élevage. Ainsi, on tient compte en partie de l'effet élevage.

où

$$
\begin{gathered}
{ }_{\theta} \mathrm{I}_{i}={ }_{\theta} \mathrm{CD}_{i} \times{ }_{\theta} \mathrm{P}_{i} \\
{ }_{\theta} \mathrm{P}_{i}=\frac{\sum_{j} \sum_{k}\left({ }_{\theta} \mathrm{X}_{i j k}-{ }_{\theta} \mathrm{X}_{. j .}\right)}{\sum_{j} n_{i_{j}}}
\end{gathered}
$$

est la moyenne des performances du mâle $i$ dans laquelle ${ }_{\theta} \mathrm{X}_{i_{j} k}$ est la performance de type $\theta$ de la $\mathrm{k}^{\mathrm{e}}$ fille du mâle $i$ dans l'élevage $j$.

${ }_{\theta} \mathrm{X}_{\text {.j. }}$ est la moyenne générale des performances de type $\theta$ des agnelles de l'élevage $j$ calculée sur des échantillons $\mathrm{A}$ ou $\mathrm{B}$.

$n_{i_{j}}$ est l'effectif de la descendance du mâle $i$ dans l'élevage $j$.

et

$$
{ }_{\theta} \mathrm{CD}_{i}=\frac{0,5 \sum_{j} n_{i_{j}{ } h^{2}}}{\mathrm{I}+\left(\sum_{j} n_{i_{j}}-\mathrm{I}\right) 0,25{ }_{\theta} h^{2}}
$$


Le coefficient de détermination ${ }_{\theta} \mathrm{CD}_{i}$ étant le coefficient de régression classiquue dans l'index de sélection sur descendance, dans lequel ${ }_{\theta} h^{2}$ est l'héritabilité du caractère considéré.

Les index " lait " utilisés sont ceux calculés selon la méthode de PoLy, PouTOUS et FREBLING (I965), par comparaison aux contemporaines de la même classe d'étable. Ils concernent la première lactation des filles et portent sur la production laitière commercialisée après 25 jours d'allaitement, production corrigée pour 1'époque de mise bas, l'âge à l'agnelage et la durée de traite. Ces index tiennent compte de la totalité des filles contrôlées sur l'ensemble des campagnes, puisque les index calculés chaque année sont cumulés aux index des années antérieures.

\section{Estimation des héritabilités}

Les héritabilités de la "prolificité naturelle " et de la " prolificité induite " sont estimées séparément sur les fichiers $C, D, E_{1}, E_{2}$ par analyse de variance et estimation du coefficient de corrélation intra-classe de père sur les données exprimées en écart à la moyenne de l'élevage.

Dans les deux cas $\theta=$ I ou 2 , le modèle mathématique de décomposition d'une performance est :

$$
\left({ }_{\theta} \mathrm{X}_{i j k}-{ }_{\theta} \mathrm{X}_{. j .}\right)={ }_{\theta} \mathrm{Y}_{i k}={ }_{\theta} \mathrm{S}_{i}+{ }_{\theta} \mathrm{E}_{k(i)}
$$

dans lequel ${ }_{\theta} \mathrm{Y}_{i k}$ est la performance corrigée de la kième fille du mâle $i$.

${ }_{\theta} \mathrm{S}_{i}$ est l'effet aléatoire du mâle $i$; nous supposons que les ${ }_{\theta} \mathrm{S}_{i}$ sont des variables aléatoires indépendantes entre elles et de loi $\mathrm{N}\left(\mathrm{O},{ }_{\theta} \sigma_{s}^{2}\right)$;

${ }_{\theta} \mathrm{E}_{k(i)}$ est l'effet résiduel propre à la $k^{\mathrm{e}}$ fille du mâle $i$; nous supposons que les ${ }_{\theta} \mathrm{E}_{k(i)}$ sont des variables aléatoires indépendantes entre elles et des ${ }_{\theta} \mathrm{S}_{i}$ et de loi $\mathrm{N}\left(0,{ }_{\theta} \sigma_{e}^{2}\right)$.

L'héritabilité est estimée égale à 4 fois le coefficient de corrélation intraclasse de père. Une estimation de son écart type est fournie par Fisher (I94I).

$$
{ }_{\theta} \sigma_{t}=(\mathrm{I}+(n-\mathrm{I}) t)(\mathrm{I}-t) \sqrt{\frac{2}{n(n-\mathrm{I})(s-2)}}
$$

dans laquelle $s$ est le nombre de mâles.

Nous avons remplacé dans cette formule $n$, nombre de filles par mâle, par le nombre moyen de filles par mâle.

\section{Estimation de la corrélation génétique}

D'après le modèle de décomposition adopté, la covariance entre les moyennes de performances d'un mâle $i$ pour les deux caractères peut s'écrire :

$$
\operatorname{cov}\left({ }_{1} \mathrm{Y}_{i .},{ }_{2} \mathrm{Y}_{i .}\right)=\operatorname{cov}\left({ }_{1} \mathrm{~S}_{i},{ }_{2} \mathrm{~S}_{i}\right)+\operatorname{cov}\left({ }_{1} \mathrm{E}_{. i},{ }_{2} \mathrm{E}_{. i}\right)=\mathrm{I} / 4 \cdot \operatorname{cov}\left({ }_{1} g,{ }_{2} g\right)
$$

Si l'on pose $\operatorname{cov}\left({ }_{1} g,{ }_{2} g\right)$ égale à la covariance génétique additive entre les caractères, et que l'on suppose $\operatorname{cov}\left({ }_{1} \mathrm{E}_{. i},{ }_{2} \mathrm{E}_{. i}\right)=0$, on peut alors écrire :

$$
\operatorname{cov}\left({ }_{1} g,{ }_{2} g\right)=4 \operatorname{cov}\left({ }_{1} Y_{. i},{ }_{2} Y . i\right)
$$


Avec les notations suivantes,

${ }_{\theta} \sigma_{g}$ : écart type génétique additif;

${ }_{\theta} \sigma_{s}^{2}$ et ${ }_{\theta}{ }_{s}^{2}$ : composante père de la variance et son estimée;

${ }_{\theta} \sigma_{\bar{p}}$ et ${ }_{\theta} \hat{\sigma}_{\bar{p}}$ : écart type phénotypique des moyennes de descendance et son estimée;

$r$ et $\hat{r}$ : corrélation entre ces moyennes et son estimée;

comme :

$$
{ }_{1} \sigma_{g}=2.1 \sigma_{s}
$$

il vient :

$$
r_{g}=\frac{\operatorname{cov}\left({ }_{1} g,{ }_{2} g\right)}{{ }_{1} \sigma_{g}{ }_{2} \sigma_{g}}=\frac{\operatorname{cov}\left({ }_{1} Y_{i_{.,}} Y_{i .}\right)}{{ }_{1} \sigma_{s_{2}} \sigma_{s}}
$$

que l'on peut estimer par

$$
\hat{r}_{g}=\frac{\hat{r}_{1} \hat{\sigma}_{\bar{p}}{ }_{2} \hat{\sigma}_{p}}{\hat{\sigma}_{s} \hat{\sigma}_{2}}
$$

\section{Résultats}

A. - Facteurs de variation non génétique de la prolificité

I. - Périodes de mise-bas et age de l'agnelage (tab1. I)

Les fécondations sur O.I. ont lieu 25 jours plus tôt en moyenne que les fécondations sur O.N. (92 p. roo des mises bas entre le r / or et le 3I /03/r977 sur O.N. entre le I6/12/1976 et le I5/02/I977 sur O.I.), mais 1'âge au premier agnelage des agnelles fécondées sur O.I. est aussi significativement plus faible que celui

TABLEAU I

Résultats zootechniques

Technical results

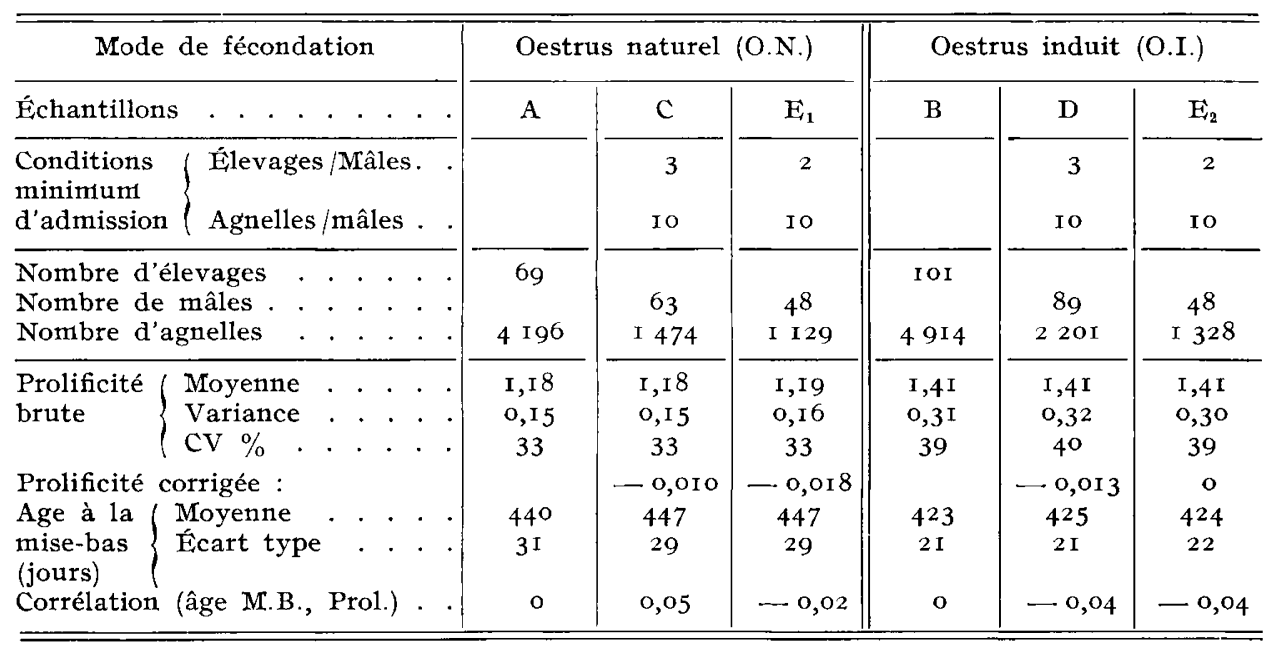


des agnelles fécondées sur O.N. : 424 contre 440 jours. Pour les deux catégories d'agnelles nous n'observons pas d'effet significatif de la quinzaine de mise-bas ou de 1'âge sur la prolificité brute, de sorte que nous n'avons pas effectué de correction pour ces deux facteurs.

\section{2. - Infuence du traitement (tabl. I)}

La prolificité obtenue sur œstrus naturel est très significativement inférieure à celle obtenue sur oestrus induit : I,I 8 contre $I, 4 \mathrm{I}$. L,es variances et coefficients de variation de ces prolificités varient dans le même sens : respectivement 0,15 et 33 p. Ioo pour O.N. et 0,30 et 39 p. roo pour O.I.

\section{3. - Différences entre élevages}

La distribution des prolificités moyennes par élevage (fig. I) est beaucoup plus étendue dans les élevages pratiquant la synchronisation (étendue de 2,00 contre 0,49 ). Il n'y a pas de corrélation entre la prolificité moyenne et l'effectif par élevage : $r=0,03$ et 0,08 respectivement pour les élevages en $0 . N$. et en O.I.

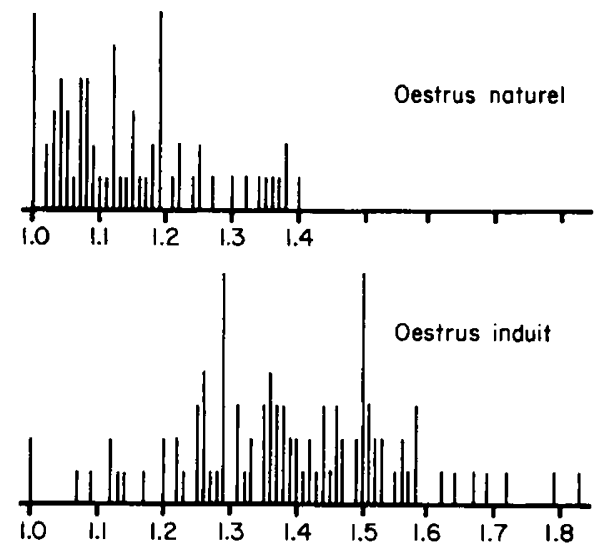

FIG. I. - Distribution de la prolificité moyenne des élevages.

Distribution of averaged prolificacy of flocks.

\section{B. - Différences phénotypiques entre descendances}

Pour supprimer en partie "l'effet élevage ", nous avons utilisé les performances corrigées, nous vérifions alors que les moyennes des prolificités par descendance sont nulles et nous constatons que la variance de la prolificité par descendance est plus élevée sur O.I. que sur O.N., soit 0,020 contre 0,009. Nous n'observons pas de corrélation significative entre la prolificité moyenne et l'effectif par descendance : $r=0$, IO et, 0, I I respectivement par O.N. et O.I., ce qui montre que la "diffusion " des mâles est indépendante de leur niveau moyen en prolificité. 


\section{C. - Index}

Parmi les I 42 béliers indexés conjointement sur le lait et la prolificité, 60 sont indexés sur O.N. et 82 sur O.I., chacun des index prolificité étant calculé sur au moins ro filles réparties dans un minimum de 3 élevages. On observe que les index "prolificité naturelle " sont moins variables que les index " prolificité induite " (les écarts type sont respectivement de 0,05 et 0,08 agneau par mise-bas) et qu'il existe une corrélation positive entre l'index " lait " d'un bélier et l'effectif de sa descendance indexée sur la prolificité en O.N. ou O.I. $(r=0,2 \mathrm{I}$ et 0,30 TS $)$ Cette corrélation peut traduire la plus grande diffusion des mâles améliorateurs en production laitière, même si cet effectif ne représente que très partiellement le nombre de filles produites, puisque la prolificité n'a été contrôlée que sur une seule campagne.

Enfin, les corrélations entre les index " lait " et " prolificité naturelle " d'une part, les index " lait " et " prolificité induite " d'autre part, sont très faibles (respectivement - 0,06 et $+0,04)$, ce qui permet de supposer que, dans notre fichier la sélection réalisée sur les performances laitières n'a pratiquement pas modifié la prolificité (tab1. 2).

TABLEAU 2

Relations entre index lait et index prolificité

Relationship between dairy index and prolificacy index

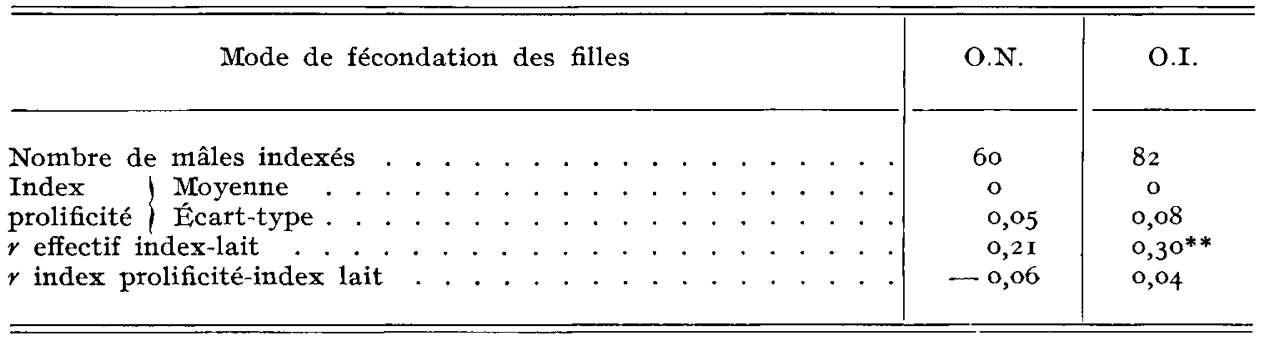

\section{D. - Héritabilités}

Le tableau 3 donne les composantes de la variance, les effectifs, les héritabilités avec les estimations de leurs écarts-type pour la prolificité sur O.N. et sur O.I. Les effectifs moyens de filles par père sont similaires dans les échantillons $\mathrm{C}, \mathrm{D}, \mathrm{E}_{1}$ et $\mathrm{E}_{2}$ et proches de l'optimum défini par RoBERTson (I959) pour avoir la variance $d u$ coefficient de corrélation intra classe minimum. $\mathrm{I}_{1}$ a variance résiduelle est plus élevée pour les agnelles synchronisées et inséminées que pour celles saillies naturellement. La composante père est faible pour les deux variables, et n'est significativement différente de zéro que pour la prolificité sur O.I. Finalement 1'héritabilité de la "prolificité induite " est légèrement supérieure à 1'héritabilité de la "prolificité naturelle ": 0,064 contre 0,032 estimée sur les échantillons C et D. On vérifie que pour les sous échantillons $\mathrm{E}_{1}$ et $\mathrm{E}_{2}$, plus réduits, ces héritabilités sont du même ordre de grandeur. 
TABLEAU 3

Composantes de la variance et héritabilité de la prolificité sur O.N. et O.I. Variance components and heritability of prolificacy with O.N. and O.I.

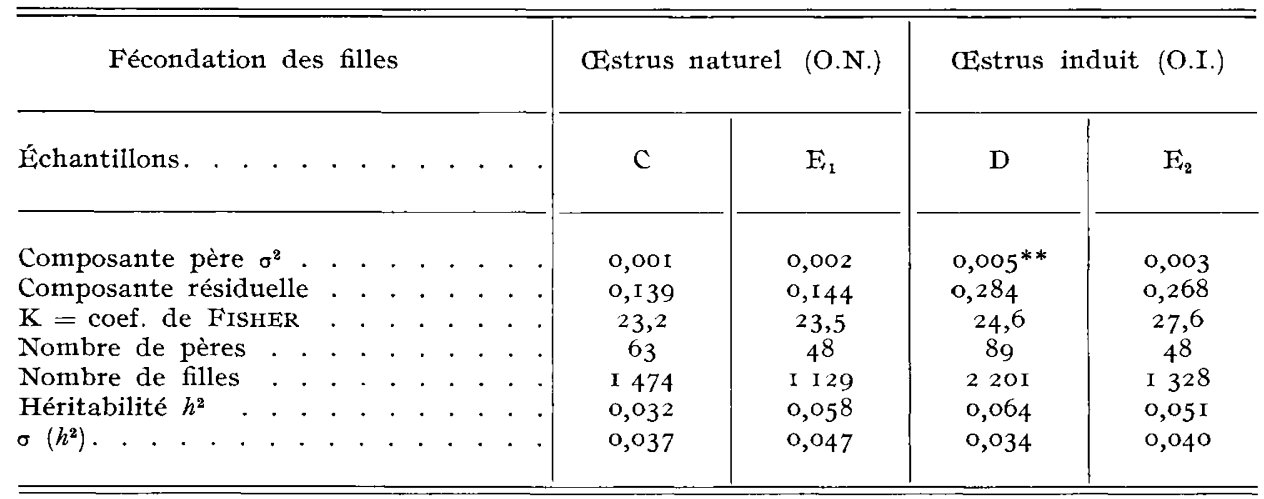

\section{E. - Corrilations}

I a corrélation entre les prolificités moyennes avec et sans traitement hormonal des filles de 48 pères des échantillons $\mathrm{E}_{1}$ et $\mathrm{E}_{\mathrm{z}}$ est $r==0,082$, les variances des moyennes de père étant $\sigma^{2}=0,0$ II 4 pour O.N. et $\sigma^{2}=0,0$ I49 pour O.I. La corrélation génétique estimée entre la " prolificité naturelle " et la "prolificité induite " est $\hat{\gamma}_{g}=0,393$, le coefficient de régression de la "prolificité induite " sur la "prolificité naturelle " étant $b=0,449$.

\section{Discussion}

I. - Hormis l'effet élevage, les autres facteurs non génétiques de variation n'ont pas d'effet significatif sur la prolificité naturelle ou induite, ce qui s'explique en partie par la standardisation des conditions d'élevage dans les troupeaux laitiers (période de lutte et âge à la mise-bas). Les variables poids à la lutte et type de naissance n'ont pas été enregistrées, ce qui nous prive d'une information intéressante sur 1'effet maternel que FALCONER (I955) a mis en évidence chez la souris. I1 est possible, en effet, qu'à 9-Io mois (âge moyen de fécondation), les agnelles nées multiples aient un poids inférieur à celles nées simples et, partant, une prolificité plus faible, comme l'a montré BEN YOUCEF (I977) en étudiant les relations prolificité-croissance sur des agnelles Lacaune de la même région. Mais l'effet défavorable du type de naissance et du mode d'allaitement risque d'être cependant limité dans notre cas, puisque la durée d'allaitement est habituellement réduite à moins de 40 jours.

2. - Nous constatons, par contre, que le traitement hormonal a un effet très hautement significatif qui se traduit par une augmentation de la taille de portée de 0,23 agneau par mise-bas. Du point de vue génétique, Matheron et Mauleon (r979) ont décomposé la taille de portée en deux composantes : I) le taux d'ovulation sous le contrôle de gènes de la femelle (effet direct) et de gènes de sa mère 
autres que les gènes d'ovulation transmis à sa fille (effet maternel); z) la viabilité des ovules fécondés dépendant de gènes propres de l'embryon (effet direct), de gènes de sa mère (effet maternel) et de gènes provenant de la mère de la femelle (effet grand materne1). Dans cette étude nous avons recherché au travers des héritabilités et de la corrélation génétique, la part des effets additifs de la prolificité considérée comme résultante de cet ensemble de composantes. Pour la " prolificité induite ", 1'héritabilité estimée est significativement supérieure à zéro alors que ce n'est pas le cas pour la " prolificité naturelle ". Cette différence pourrait être due à une action plus importante des effets maternels sur la "prolificité naturelle "que sur la " prolificité induite " pour laquelle le traitement hormonal diminue ces effets en standardisant plus l'état physiologique des agnelles. Nos résultats sont comparables à ceux des études antérieures en ce qui concerne la prolificité naturelle (RICORDEAU et al., I979); ils concordent bien avec ceux obtenus par GABIÑA (I979) qui analyse les performances des agnelles Lacaune " lait " de trcis campagnes successives, dans des élevages affiliés à l'un des organismes de sélection.

La différence observée entre les héritabilités tend à montrer que les deux prolificités ne sont pas des caractères identiques; comme la dose de PMSG est relativement faible, on peut supposer que le traitement hormonal ne modifie pas de façon sensible le pourcentage de pertes embryonnaires des ovules obtenus sur O.N. et O.I., de sorte que les différences entre prolificités naturelle et induite doivent correspondre aux différences entre ovulation naturelle et ovulation induite. Dans cette hypothèse nos résultats peuvent être comparés à ceux de IAND et FALCONER (I 969) qui, sur souris, observent entre les deux caractères taux d'ovulation, une corrélation génétique égale à 0,33 (moyenne des estimations faites à partir des réponses indirectes à la sélection : 0,22 pour sélection en $O . N$. et 0,46 pour sélection en O.I.). Cela suppose donc que les taux d'ovulation naturelle et induite sont des caractères contrôlés en partie par des gènes différents. Cette conclusion mériterait d'être approfondie du point de vue physiologique. En effet, comme le taux d'ovulation peut se décomposer en activité $\mathrm{FSH}$ endogène et sensibilité ovarienne (MATHERON et MAULEON, I979), il serait intéressant - après avoir précisé les variabilités du taux d'ovulation et de de la mortalité embryonnaire - d'analyser certaines descendances comparables en prolificité naturelle mais très différentes en prolificité induite et inversement.

3. - Notre étude ne prétend pas donner une estimation précise de la corrélation génétique entre les prolificités avec et sans traitement hormonal. En effet, sa précision dépend du nombre de descendances, des effectifs par descendance et de la répartition des filles dans les différents élevages. Dans notre fichier, cette répartition est particulière puisque les filles avec ou sans synchronisation sont dans des élevages différents, ce qui risque d'apporter une confusion partielle des effets élevage, père et traitement, lors de 1'analyse. On doit aussi émettre des réserves au sujet de la signification de la corrélation génétique, compte tenu de l'existence d'un infiniment petit au dénominateur (composante de la variance non différente de zéro).

Bien qu'étant assez imprécise, la corrélation génétique entre prolificité naturelle et prolificité induite peut nous donner une indication de la réponse en prolificité naturelle si l'on sélectionne sur la prolificité induite. Le rapport de la réponse corrélée $\mathrm{RC}_{\mathrm{ON}}$ à la réponse directe $\mathrm{R}_{\mathrm{ON}}$

$$
\frac{\mathrm{RC}_{\mathrm{ON}}}{\mathrm{R}_{\mathrm{ON}}}=r_{g} \frac{h_{\mathrm{OI}}}{h_{\mathrm{ON}}}=0,55 \text { indique que, toutes choses étant égales par ailleurs, }
$$


la sélection directe pour augmenter la prolificité naturelle en première mise-bas est plus efficace que la sélection indirecte au travers de la prolificité induite. Mais à l'échelle d'une race le problème se pose en termes plus complexes, compte tenu des avantages qu'apporte le traitement hormonal pour l'éleveur et le sélectionneur. I1 faut en effet distinguer la mise en place du testage, le testage proprement dit et la diffusion du progrès génétique. Le tableau 4 résume, pour chacun de ces postes, les avantages consécutifs à la synchronisation des cestrus, à l'insémination artificielle et au gain de prolificité obtenu.

\section{TABLEAU 4}

Répartition des avantages de la synchronisation, de l'insémination des gains de prolificité selon 3 postes d'un schéma de sélection

Distribution of advantages of synchronisation, insemination and increase of prolificacy level at three stages of a selection scheme

\begin{tabular}{|c|c|c|c|c|}
\hline & Avantages & $\begin{array}{l}\text { Syn- } \\
\text { chro }\end{array}$ & I.A. & $\begin{array}{l}\text { Gain } \\
\text { de } \\
\text { prol. }\end{array}$ \\
\hline $\begin{array}{c}\text { Mise en place } \\
\text { du testage }\end{array}$ & $\begin{array}{l}\text { - Répartition des mâles dans les élevages. } \\
\text { Programmation des accouplements des brebis } \\
\text { support. } \\
\text { - Nombre de filles contemporaines } \\
\text { - }\end{array}$ & $\star$ & $\star$ & $\star$ \\
\hline Testage & $\begin{array}{l}\text { - Obtention de cohortes homogènes. } \\
\text { - Réduction des effets du milieu . . . . . . . . . } \\
\text { - Diminution de l'intervalle entre performances }\end{array}$ & $\begin{array}{l}\star \\
\star \\
\star\end{array}$ & & \\
\hline $\begin{array}{l}\text { Diffusion } \\
\text { du progrès } \\
\text { génétique }\end{array}$ & $\begin{array}{l}\text { - Pression de sélection. } \\
\text { - Diminution de l'intervalle de génération. } \\
\text { - Diffusion des améliorateurs } . . . \\
\end{array}$ & $\star$ & $\star$ & $\star$ \\
\hline
\end{tabular}

Pour des races dont la prolificité est l'objectif de sélection prioritaire on peut distinguer schématiquement trois situations :

a) Pour des raisons zootechniques ou économiques, les traitements hormonaux ne sont pratiquement pas utilisés par les éleveurs et les sélectionneurs (par exemple schéma d'amélioration de la race Aragonaise: GABIÑa et BoDIn, r979). Dans ce cas, la sélection tient compte uniquement des performances obtenues sur O.N. tout en sachant que le testage sur descendance est difficile à mettre en place.

b) Les traitements hormonaux sont utilisés sur les brebis de plus de deux ans, uniquement pour la mise en place du testage des béliers. Ils servent à produire le nombre suffisant de filles par père dans plusieurs élevages. Dans ce cas, le nombre de brebis soumises au traitement hormonal peut être important $(25 \mathrm{p} .100$ minimum pour un troupeau de $\mathrm{r} 2 \mathrm{O}$ femelles), notamment si la diffusion se fait par insémination. Ce qui oblige à prendre en compte la prolificité sur O.I. dans les objectifs de sélection, même si ces performances ne sont pas nécessaires à l'estimation de la valeur génétique des mâles. 
c) Suivant les époques, les brebis sont synchronisées et inséminées, ou saillies naturellement. Il s'agit alors de maximiser le progrès à la fois sur O.N. et O.I. en exprimant la valeur génétique $\mathrm{G}_{i}$ du mâle par un index

$$
\mathrm{G}_{i}=\mathrm{I} / 2\left({ }_{\mathrm{oN}} \mathrm{E}_{i} \times{ }_{\text {oN }} g_{i}+{ }_{\text {or }} \mathrm{E}_{i} \times \text { org }_{i}\right)
$$

dans lequel, on $g_{i}$ et or $g_{i}$ sont les valeurs génétiques du mâle pour ces deux caractères on $\mathrm{E}_{i}$ et ${ }_{\text {oI }} \mathrm{E}_{i}$ des coefficients de pondération économiques et zootechniques, le coefficient I / 2 étant dû au fait que la prolificité ne s'exprime que chez les descendances des mâles.

Quelle que soit 1a situation, l'objectif est d'améliorer la prolificité des brebis sur l'ensemble de leur carrière, ce qui suppose la connaissance de la valeur prédictrice des performances obtenues en première mise-bas sur les performances suivantes, ou des deux ou trois premiers agnelages sur 1'ensemble de la carrière. Or, nous savons que les estimations de la répétabilité de la taille de portée sont souvent faibles et que le taux d'ovulation naturel des nullipares n'est peut-être pas le même caractère que celui des primipares contrairement au taux d'ovulation induit : LAND et FALCONER (I969) constatent en effet que la corrélation génétique entre primipares et nullipares est seulement de 0,46 dans le cas d'ovulation naturelle contre 0,82 dans le cas d'ovulation induite.

D'un point de vue général, il serait donc souhaitable de vérifier certaines des hypothèses génétiques que nous avons soulevées et de préciser les premières estimations des corrélations gérétiques calculées dans cette étude, afin de définir une meilleure stratégie de la sélection des ovins sur la taille de portée.

\section{Reçu pour publication en février 1980.}

\section{Summary}

\section{Estimation of the genetic parameters of Lacaune ewe lamb size after fecundation on natureel litte and induced astrus}

Genetic parameters of litter size of 9 I I Lacaune ewe lambs from I7o milk flocks were analyzed. Fecondations were made on natural œstrus or œstrus induced by vaginal sponge impregnated with F.G.A. and injection of PMSG. "Induced prolificacy " $(\mathrm{I}, 4 \mathrm{I})$ is very significantly higher than "natural prolificacy". Effects of lambing age and mating season are not significant.

Heritability of litter size estimated by half-sib variance analysis is significantly different from o for " induced prolificacy" $\left(h^{2}=0,064\right)$ but not for " natural prolificacy" $\left(h^{2}=0,032\right)$. Moreover, genetic correlation between these traits $\left(v_{g}=0,39\right)$ let suppose that natural and induced prolificacy are partially controled by the same genes.

Finally, three classical situations for the use of hormonal treatment in French structures are presented and its implications of selection schemes for reproductive traits are indicated.

\section{Références bibliographiques}

BEnyoucef M. T., I977. Contrôle de Performances et analyse de la variabilité génétique chez les Ovins. Thèse $3^{\mathrm{e}}$ cycle I.N.P., Toulouse.

Bodin L., Ricordeau G., Tchamitchian I., PoujardieU B., r979. Variabilité génétique des performances de reproduction des agnelles Lacaune, avec et sans traitement hormonal de synchronisation des œstrus. Symposium sur la reproduction des bovins et des ovins de boucherie. Mai I979, Saragosse. 
Colas G., Thimonier J., Courot M. et OrTavant R., i973. Fertilité, prolificité et fécondité pendant la saison sexuelle des brebis inséminées artificiellement après traitement à l'acétate de fluorogestone. Ann. Zootech., 22, 44I-45I.

FALCONER D. S., I955. Patterns of response in selection experiments with mice. Cold Spr. Harb. Symp. quant. Biol., 20, 178-196.

FISHER R. A., I94I. Statistical methods for research workers. $8^{\mathrm{e}}$ éd. xiii +399 pp., Edimburgh: OLIVER and BOYD.

FoUl.LEY J. L., BIBE B., I979. Utilisation conjointe de l'I.A. et de la saillie naturelle en vue de $1^{\prime}$ indexation des reproducteurs. In $5^{\mathrm{e}}$ journées de la Recherche Ovine et Caprine. Paris, décembre 1979. Ed. Itovic S.P.E.O.C.

GABIÑa D., I979. Estudio genetico de la prolificidad obtenida como repuesta a tratamientos hormonales en ganado ovino. Thesis doctoral, Escuela Tecnica Superior de Ingenieros Agronomos de Madrid.

GABIÑA D., BoDIN L., I979. Organizacion de la Seleccion en la rasa Aragonesa. IV Jornadas cientificas de 1a Sociedad Espanola de Ovinotecnia.

LAND R. B., FALCONER D. S., I969. Genetic studies of ovulation rate in the mouse. Genet. Res., 13, 25-46.

MathERON G., MaUleion P., I979. Mise en évidence de l'actions conjointe des effets directs maternels et grands maternels sur la taille de portée. Bull. Tech. Dept. Génét. Anim., (29-30), 423-424.

Pol,y J., Poutous M., Frebling J., I965. Méthode de calcul des index de production laitière. Bull. Tech. inf. (205), 957-964.

Ricordeau G., Bodin L., Tchamitchian L., I979. Amélioration de la prolificité des brebis par sélection. Revue bibliographique. In $5^{\mathrm{e}}$ journées de la Recherche Ovine et Caprine. Paris, décembre I979. Ed. ITOVIC-S.P.E.O.C.

ROBERTSON A., I959. Experimental design in the evaluation of genetic parameters. Biometrics, 15, $219-226$.

Thimonier J., Mauleon P., Cognie Y., Ortavant R., ig68. Déclenchement de l'œstrus et obtention précoce de gestations chez les agnelles à l'aide d'éponges vaginales imprégnées d'acétate de fluorogestone. Ann. Zootech., 17, 275-288. 